\title{
Peculiar emission from the new VHE gamma-ray source $\mathrm{H} 1722+119$
}

\section{T. Terzić ${ }^{1}$, A. Stamerra ${ }^{2}$, the MAGIC Coll. ${ }^{3}$, F. D'Ammando ${ }^{4,5}$ (for the Fermi-LAT Coll.), C. M. Raiteri ${ }^{6}$, M. Villata ${ }^{6}$, F. Verrecchia $^{7,8}$ and O. Kurtanidze ${ }^{9}$}

\begin{abstract}
${ }^{1}$ University of Rijeka, 51000 Rijeka, Croatia, email: tterzic@phy.uniri.hr; ${ }^{2}$ INAF National Institute for Astrophysics, I-00136 Rome, Italy; ${ }^{3}$ The full list of collaborators can be found at: wwwmagic.mppmu.mpg.de; ${ }^{4}$ Dip. Di Fisica e Astronomia, Universit degli Studi di Bologna, I-40127 Bologna, Italy; ${ }^{5}$ INAF Istituto di Radioastronomia, I-40129 Bologna, Italy; ${ }^{6}$ INAF Osservatorio Astrofisico di Torino, I-10025 Pino Torinese (TO), Italy; ${ }^{7}$ INAF Osservatorio Astronomico di Roma, I-00040 Monteporzio Catone, Italy; ${ }^{8}$ ASI Science Data Center (ASDC), I-00133 Roma, Italy; ${ }^{9}$ Abastumani Observatory, Mt. Kanobili, 0301 Abastumani, Georgia
\end{abstract}

Keywords. BL Lacertae objects: individual (H1722+119), gamma-rays, multiwavelength

The BL Lac object H1722+119 was observed in the very high energy band (VHE, $E>$ $100 \mathrm{GeV}$ ) by the MAGIC (Major Atmospheric Gamma-ray Imaging Cherenkov) telescopes (Aleksić et al. 2016a, b) between 2013 May 17 and 22, following a state of high activity in the optical band measured by the KVA (Kungliga Vetenskapsakademien $\dagger$ ) telescope. Optical high states are often used to trigger MAGIC observations, which result in the VHE $\gamma$-ray signal detection (see e.g. Aleksić et al. 2015, Ahnen et al. 2016 and references therein).

Integrating $12.5 \mathrm{~h}$ of observation, the source was detected at VHE with a statistical significance of 5.9 sigma. The flux appears to be constant at the level of $f=(6.3 \pm 1.6) \times 10^{-12} \mathrm{ph} \mathrm{cm}^{-2} \mathrm{~s}^{-1}$ above $150 \mathrm{GeV}$ with $\chi^{2} / N_{\text {d.o.f. }}=3.5 / 5$ (fig. 1 right top). This corresponds to $(2.0 \pm 0.5) \%$ of the Crab Nebula flux in the same energy range. Contemporaneous observations were performed by the Fermi-LAT (Large Area Telescope, Atwood et al. 2009, Ackermann et al. 2012) in the high energy range (HE, $100 \mathrm{MeV}<E<100 \mathrm{GeV}$ ); by the Swift-XRT: $200 \mathrm{eV}<E<10 \mathrm{keV}$ (Burrows et al. 2005) and the Swift-UVOT: $170 \mathrm{~nm}<\lambda<600 \mathrm{~nm}$ (Roming et al. 2005); by the KVA and the Abastumani telescope $\ddagger$ in the $R$-band, and by the OVRO (Owens Valley Radio Observatory) telescope in the $15 \mathrm{GHz}$ radio band (Richards et al. 2011).

The multiwavelength light curve (fig. 1 left) shows no significant temporal variability in the HE band either. The flux was $f=(3.4 \pm 0.6) \times 10^{-8} \mathrm{ph} \mathrm{cm}^{-2} \mathrm{~s}^{-1}$, with $\chi^{2} / N_{\text {d.o.f. }}=3.9 / 5$, while the spectrum appears to be constant as well, with the spectral index of $1.96 \pm 0.07$, with $\chi^{2} / N_{\text {d.o.f. }}=1.7 / 5$. The optical flux varied with no apparent regularity, and the radio data show a linear trend $\left(F[\mathrm{Jy}]=p_{0}+p_{1} \times(t-56300)[\mathrm{MJD}]\right)$ of increasing flux on a time-scale of one year, with the fit parameters $p_{0}=(5.2 \pm 0.2) \times 10^{-2} \mathrm{Jy}, p_{1}=(1.07 \pm 0.10) \times 10^{-4} \mathrm{Jy} /$ day and $\chi^{2} / N_{\text {d.o.f. }}=39.68 / 36$.

Using the HE and VHE gamma-ray observations, and the expected imprint of the extragalactic background light (EBL, Franceschini et al. (2008)) absorption, we estimated the redshift of the source to be $z=0.34 \pm 0.15_{\text {stat }} \pm 0.05_{\text {meth }}$ with upper limit (UL) of $z<1.06$ (95\% C.L.), using the method from Prandini et al. (2010). Using the method of Mazin \& Goebel (2007) we obtain an UL of $z<0.95$. Our reconstructed redshift value is in agreement with the latest Landoni et al. (2014) and Farina et al. (2013, priv. comm.) results. A quasi simultaneous multi-wavelength SED (spectral energy distribution, fig. 1 right bottom) shows an unexpected feature in the $3 \times 10^{14}-10^{18} \mathrm{~Hz}$ frequency range. A possible explanation using an inhomogeneous helical jet synchrotron self-Compton model is suggested (Villata \& Raiteri 1999, Ahnen et al. 2016).

$\dagger$ http://users.utu.fi/kani/1m

$\ddagger$ http://www.oato.inaf.it/blazars/webt/aba_info.html 
$2013 / 02 / 09 \quad 2013 / 04 / 07 \quad 2013 / 06 / 04 \quad 2013 / 08 / 01 \quad 2013 / 09 / 28 \quad 2013 / 11 / 25$

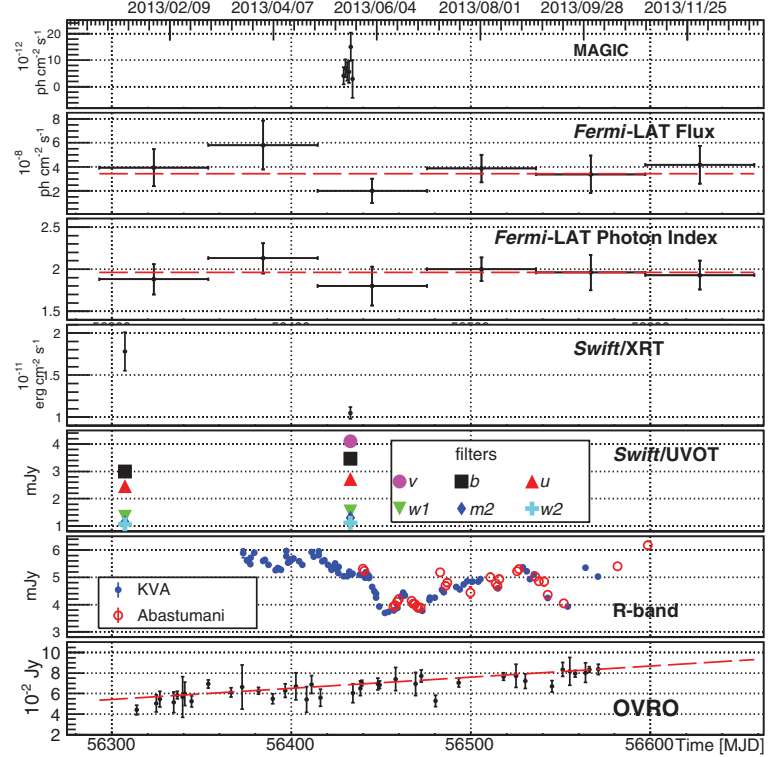

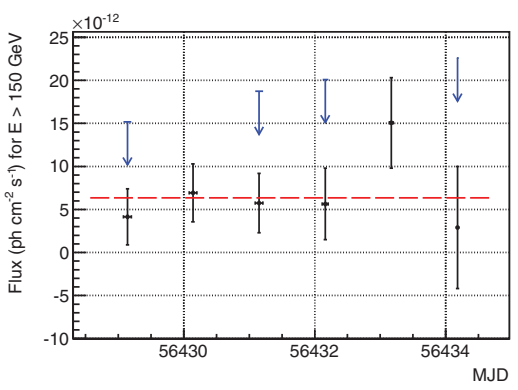

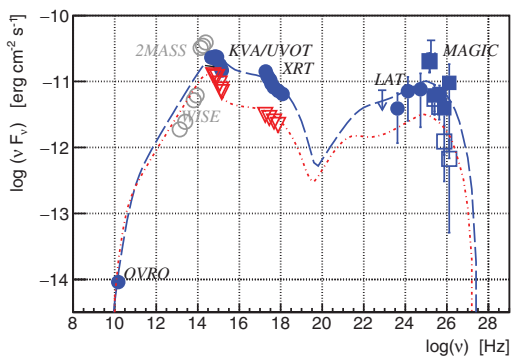

Figure 1. Light curve and the SED of H1722+119 for data collected during 2013. Left: Multiwavelength light curve during 2013. The data were collected (from top to bottom) by MAGIC, Fermi-LAT, Swift/XRT, Swift/UVOT, KVA and Abastumani and OVRO. The HE $\gamma$-ray light curve and the evolution of the spectral index, as measured by Fermi-LAT, are shown in 2-month time bins, while daily observations time-scale is used in the light curves in the other energy bands. Right top: MAGIC nightly light curve for energies above $150 \mathrm{GeV}$. Horizontal error bars represent the duration of each observation. Vertical arrows represent ULs for points whose relative error of the excess is $>0.5$. Right bottom: The H1722+119 SED. Full circles represent data contemporaneous to MAGIC observations. The MAGIC measured data are shown by empty squares, while full squares indicate the de-absorbed points. Fermi-LAT data are shown by full circles, while arrows represent Fermi-LAT ULs. The Swift data indicated by full circles were taken on 2013 May 20 (MJD 56432). The KVA $R$-band point represented by a full circle was taken on the same night. The OVRO measurement represented by a full circle was taken on 2013 May 22 (MJD 56434). Empty triangles show the Swift data from 2008 May 31 (MJD 54617). The solid line in the $10^{14}-10^{15} \mathrm{~Hz}$ range indicating data from Landoni et al. (2014) was not considered for modelling, as well as archival data from 2MASS and WISE shown by empty circles. The long-dashed line indicates the fit of the helical jet model to the 2013 data, while the fit to the 2008 data is indicated by the dash-dot line. Both models represent the intrinsic VHE emission.

\section{References}

Ackermann, M., et al. 2012, ApJS, 203, 4

Ahnen M. L., et al. 2016, MNRAS, 459, 3271

Aleksić J., et al., 2015, MNRAS, 451, 739

Aleksić J., et al. 2016a, Astroparticle Physics, 72, 61

Aleksić J., et al. 2016b, Astroparticle Physics, 72, 76

Atwood W. B., et al. 2009, ApJ, 697, 1071

Burrows D. N., et al. 2005, Space Sci. Rev., 120, 165

Franceschini, A., Rodighiero, G., \& Vaccari M. 2008, A\&A, 487, 837

Landoni, M., et al. 2014, A\&A, 570, A126

Mazin, D., Goebel F. 2007, ApJ, 655, L13

Prandini, E., et al. 2010, MNRAS, 405, L76

Richards J. L., et al. 2011, ApJS, 194, 29

Roming, P., et al. 2005, Space Sci. Rev., 120, 95

Villata, M. \& Raiteri, C. M. 1999, A\&A, 347, 30 